\title{
Computer software for understanding resonances and resonance-related phenomena in chemical reactions
}

\author{
Dmitri Sokolovski ${ }^{1,3}$ and Elena Akhmatskaya ${ }^{2,3}$ \\ 1 Departmento de Química-Física, Universidad del País Vasco, UPV/EHU, Leioa, \\ Spain \\ 2 Basque Center for Applied Mathematics (BCAM), Alameda de Mazarredo, 14 \\ 48009 Bilbao, Bizkaia, Spain \\ 3 IKERBASQUE, Basque Foundation for Science, E-48011 Bilbao, Spain
}

\section{References}

\begin{abstract}
In numerical modelling of chemical reactions one calculates the scattering matrix for the required values of energy and angular momentum. Having done so, one still faces the non-trivial task of extracting detailed information about the reaction mechanism. We discuss the methods and numerical tools for such an analysis in terms of resonance poles and semiclassical trajectories. Our approach avoids calculating the scattering matrix in semiclassical approximation, and employs its numerical values computed previously by an accurate scattering code.
\end{abstract}

Keywords: chemical reactions, complex angular momentum analysis, semiclassical methods

\section{Introduction}

An atom-diatom chemical reaction is a process in which an atom $A$ collides with a molecule $B C$, in such a way that after the collision the atom $B$ goes with $A$, forming a new molecule $A B$. Numerical modelling of such a recation involves at least three principal steps.

As a first step, we require the forces acting on all collision partners or, equivalently the potential energy as a function of their positions. This is a task for quantum chemists who calculate the potential energy surface (PES) using the fact that the light fast electrons adjust their motion to the movement of slow heavy nuclei. The adiabatic theorem ensures that electrons stay in the same quantum states, and absorb or return energy to the nuclear motion.

In the second step, we need to calculate the dynamical quantities, that is to solve the equations of motion for the nuclei, in order to find out whether in the end the atoms $A$ and $B$ do indeed stick together. These can be done in various ways, and at different levels of accuracy. We can just solve the threeparticle Schroedinger equation (SE) numerically, obtaining a full quantum solution, whose accuracy is only limited by the accuracy of the PES. There are many computer codes which can perform this task well (see, for example, 1-3). 
The third step consists in understanding the results, and some of its aspects are the subject of this paper. We may feel that something is still missing: atoms are heavy particles, and we are right to expect them to behave in an almost (semi-) classical fashion. The simplest way to do so is to treat them as little balls moving under known forces, and solve the corresponding Newton equations. This is, in essence, the quasiclassical trajectories (QCT) method, successfully used by many authors (see, e.g., Ref.4). But then we lose the interference effects and, among them, some of the resonance phenomena.

A resonance mechanism usually implies a process in which the reactants temporarily form a triatomic complex, or quasi-molecule, $A B C$, which after some time breaks up into fragments $A B$ and $C$. If that happens, both the total reaction cross section and the angular distributions can be affected in various ways. Formation of such complexes is usually possible at relatively low energies, and can become the dominant process, for example, in the cold conditions of the early Universe.

To recover the interference effects we may try to equip each classical trajectory with the corresponding probability amplitude, and add the amplitudes according to the laws of quantum mechanics. This approach was pioneered in the early 70 's of the last century 5 , and progressed considerably since then 6 . It does, however, meet with considerable technical difficulties, and it is probably fair to say that solving the exact SE numerically using a standard code is often an easier choice. This brings us to the main subject of this paper. Since solving the SE semiclassically is difficult, we first obtain an exact numerical solution, and then extract from it the essential semiclassical physics, the information about the scattering mechanisms involved, and explanations for various interference patterns which may appear. Like the first two steps described above, such analysis of numerical data requires computer software. In the next Section we will try to be more specific about some of the tasks to be performed by these codes.

\section{The task of interpreting numerical results}

A typical time independent computer code 1, 3, employed to model the dynamics of the atoms involved, evaluates the probability amplitudes for the reactants prepared in some quantum state $\nu$ to end up as products in some final state $\nu^{\prime}$. These amplitudes are given by complex valued scattering $(S-)$ matrix elements,

$$
S_{\nu^{\prime} \leftarrow \nu}^{\alpha}(E, J)=\left|S_{\nu^{\prime} \leftarrow \nu}^{\alpha}\right| \exp \left[i \Phi_{\nu^{\prime} \leftarrow \nu}^{\alpha}\right],
$$

evaluated as functions of two conserved quantities, the energy $E$, and the total angular momentum at discrete values $J=0,1,2 \ldots$ (we use $\hbar=1$ ). The superscript $\alpha$ tells us what has happened: if no reaction occurs, $A+B C \rightarrow A+B C$, $\alpha=0$ for an elastic event in which the state of the products is unchanged, i.e., $\nu=\nu^{\prime}$, and $\alpha=1$ if $\nu \neq \nu^{\prime}$. For a reaction, $A+B C \rightarrow A B+C$, we put $\alpha=2$. For an atom-diatom collision the index $\nu=(v, j, \Omega)$ includes vibrational and rotational quantum numbers of the diatomic, $v$ and $j$, and the projection of the diatomic's angular momentum onto atom-diatom relative velocity, $\Omega$, also 
known as helicity. Together $(v, j, \Omega)$ and $\left(v^{\prime}, j^{\prime}, \Omega^{\prime}\right)$ completely determine the states of the system before and after the collision, as long as we do not consider the possibility of its breakup, $A+B C \rightarrow A+B+C$.

Squaring the amplitudes (1), one gets the probabilities, $P_{\nu^{\prime} \leftarrow \nu}^{\alpha}(E, J)=\left|S_{\nu^{\prime} \leftarrow \nu}^{\alpha}\right|^{2}$, for the system prepared in the state $\nu$ to go to the state $\nu^{\prime}, \alpha$, as a result of a collision. These are not, however, the probabilities directly observed in an experiment where the atoms bombard a molecule with all possible angular momenta. There the two quantities usually measured are the integral (ICS) and the differential (DCS) cross sections. An ICS $\sigma_{\nu^{\prime} \leftarrow \nu}^{\alpha}(E)$ is the total probability that a transition $\nu \rightarrow \nu^{\prime}, \alpha$ will take place, regardless of where the products are scattered as a result. It is given by the expression

$$
\sigma_{\nu^{\prime} \leftarrow \nu}^{\alpha}(E)=\frac{2 \pi}{k_{\nu}^{2}} \sum_{J=J_{\min }}^{\infty}(J+1 / 2)\left|S_{\nu^{\prime} \leftarrow \nu}^{\alpha}(E, J)-\delta_{\alpha 0} \delta_{\nu \nu^{\prime}}\right|^{2},
$$

where $\delta_{i j}$ is the Kronecker delta, $k_{\nu}$ is the reactant's relative wave vector, and $J_{\min }=\max \left(|\Omega|,\left|\Omega^{\prime}\right|\right)$.

A DCS $\sigma_{\nu^{\prime} \leftarrow \nu}^{\alpha}(E, \theta)$ gives the probability that the products would fly apart in the direction making an angle $\theta$ with the initial velocity of the reactants, so that summing the DCS over all angles gives the ICS in Eq.(2). It is written as $\sigma_{\nu^{\prime} \leftarrow \nu}^{\alpha}(E, \theta)=\left|f_{\nu^{\prime} \leftarrow \nu}^{\alpha}(E, \theta)\right|^{2}$, where $f_{\nu^{\prime} \leftarrow \nu}^{\alpha}(E, \theta)$ is the corresponding probability amplitude,

$$
f_{\nu^{\prime} \leftarrow \nu}^{\alpha}(E, \theta)=\frac{1}{i k_{\nu}} \sum_{J=J_{m i n}}^{\infty}(J+1 / 2) d_{\Omega \Omega^{\prime}}^{J}(\cos \theta)\left[S_{\nu^{\prime} \leftarrow \nu}^{\alpha}(E, J)-\delta_{\alpha 0} \delta_{\nu \nu^{\prime}}\right] .
$$

Here $d_{\Omega \Omega^{\prime}}^{J}$ is a reduced rotation matrix elements (for details see, e.g., 7 and Refs. therein).

Inserting the computed values of the $S$-matrix elements (1) into the general formulae (2) and (3) certainly gives the correct answers for the cross sections. Yet it contributes little to the semiclassical picture in which atoms follow their trajectories, and may all stick together for a while before falling apart again. And without such picture, we argued, our understanding of what happens in a reaction is incomplete. The DCS (3) and ICS (2) are often structured, and the structures may point to a particular reaction mechanism we want to understand. We would like to say, for example, "these peaks, or oscillations, in the ICS happen because the reactants tend to stay together for a while, before parting as products". Or "these oscillations in the DCS occur because the system can go into this angle $\theta$ in two different ways, either directly, or by forming a quasimolecule first, and then letting it rotate for a while". To be able to say this, we require different representations for the ICS and DCS, and, ultimately, need to know more about the $S$-matrix element itself.

\section{Trajectories vs. poles of the $S$-matrix}

The simplest system one can consider is the one in which there is only one classical trajectory leading to each angle $\theta$. In such a system the interference effects are 
minimal, and it should be well modelled by the QCT technique mentioned above. We can arrive at the idea of trajectories in the following way. First we replace the sum in Eq.(3) by an integral, or integrals over $J$. This can be done exactly, for example by using the Poisson sum formula 8 . Then we replace the $d$-matrices by their asymptotic forms for large $J$ 's, which are the $J$ 's that contribute most, since the system is semiclassical. Evaluating resulting integrals by the stationary phase (SP) method establishes one-to-one correspondence between an angle $\theta$ and the angular momentum $J(\theta)$ which scatters into it. We can consider a more complex case, where there are two or more values of $J$ leading to the same angle $\theta$. Summing the contributions from all such $J$ 's and squaring the module might explain why the DCS has an oscillatory pattern. We will not elaborate on the subject. Note, however, that an analysis of this type requires the knowledge of the $S$-matrix element for all values of $J$, while a typical computer code only produces its values for the integer 'physical' values $J=0,1,2, \ldots$.

Another language, often used to describe resonances, involves the poles of the $S$-matrix element. There are two types of poles. Of one type are the discrete complex values of energy $E_{n}(J), n=1,2, .$. , for which $S_{\nu^{\prime} \leftarrow \nu}^{\alpha}$ becomes infinite for a given value of $J, S_{\nu^{\prime} \leftarrow \nu}^{\alpha}\left(E_{n}, J\right)=\infty$. These complex energy (CE) poles have their counterparts, discrete complex values of the angular momentum (Regge poles) $10, J_{n}(E), n=1,2, .$. , for which $S_{\nu^{\prime} \leftarrow \nu}^{\alpha}$ becomes infinite for a given value of $E, S_{\nu^{\prime} \leftarrow \nu}^{\alpha}\left(E, J_{n}\right)=\infty$. Like the CE poles, Regge poles may lie on the real axis only if they correspond to the bound states supported by the potential. The two kinds of poles are closely related: one can think of a function $\mathcal{F}(E, J)$ such that solving the equation

$$
\mathcal{F}(E, J)=0
$$

for $E$ as a function of $J$ one gets the positions of the CE poles $E(J)$, while solving it for $J$ yields those of the Regge poles $J(E) 9,11,12$. The fact that both $E(J)$ and $J(E)$ are typically single valued on a multi-sheet Riemann surface, explains why there are many $\mathrm{CE}$ poles for a given value of $J$, and also many Regge poles for a given $E$. Of the two kinds of poles, Regge poles are better suited for our purposes. Replacing, as discussed above, the sums in Eqs.(2) and (3) with integrals over $J$, we are able to deform the contour of integration, and separate the pole contributions to both the DCS and ICS, while keeping $E$ fixed. The CE poles would be best employed, if we were studying time delay of a wave packet, where we would need to integrate over the energy, and not the angular momentum. This is, however, a different topic.

Relation between poles and trajectories is a subject of an ongoing debate 13, and is worth a brief discussion. It is reasonably to assume (we are not aware of a rigorous proof) that the knowledge of all poles and branch cuts of the $S$ matrix in the complex $J$-plane, together with the knowledge of its behaviour as $|J| \rightarrow \infty$ would amount to knowing all dynamical properties of the system. Then the simple system mentioned at the beginning of this Section can equally be described in terms of trajectories, or in terms of poles. The pole description would, however, be awkward. To see how a large number of Regge poles, located 
far from the real $J$-axis, conspire to produce the effects of a single classical trajectory, we would need to accurately know their positions and residues. In this case it is much easier to speak about trajectories.

There is, however, a counter example. Consider the simple potential model studied in Refs.12,14,15 where most of the trajectories bounce off the outer edge of the potential. There is also a sharp resonance, so that the particles with $J \approx J_{1}$ can penetrate the barrier by tunnelling and become trapped in the interior for a significant length of time. Here full description in terms of trajectories is not possible, and the trapped motion needs to be described in terms of poles.

Between these two extremes, there are numerous cases which can be described in both languages. Such are some of the rainbows 14,16, glories 17,16 and closely related threshold phenomena 14,18. The choice of the description is in these cases voluntary.

It seems desirable to avoid discussions where one party labels a phenomenon a resonance, since it can be described in terms of poles, while the other party denies that, since there is a signature of the phenomenon in the classical trajectories simulations. With this in mind, we introduced the term 'resonance-related' in the title, and will use it wherever a similar ambiguity might arise.

Analysis in terms of poles requires the knowledge of analytical properties of the $S$-matrix element off the real $J$-aixs. In the next Section we describe a code designed to perform analytical continuation of $S_{\nu^{\prime} \leftarrow \nu}^{\alpha}(J)$ into the complex $J$-plane.

\section{Padé reconstruction of a scattering matrix element. The PADE_II code}

So we have used one of the standard codes to evaluate numerically $N+1-J_{\min }$ values of the $S$-matrix element for a transition $\nu \rightarrow \nu^{\prime}, \alpha$,

$$
S_{\nu^{\prime} \leftarrow \nu}^{\alpha}(E, J=i), \quad i=J_{\min }, \ldots, N .
$$

The number of angular momenta has been chosen large enough, so that in the partial wave sums (PWS) we can replace the infinite upper limit with $N$. Both energy $E$ and angular momentum $J$ enter the Schroedinger equation in such a way, that $S_{\nu^{\prime} \leftarrow \nu}^{\alpha}(E, J)$ is an analytical function of $E$ and $J$. It can be defined for all $J$ 's, real or complex, and not just for the integer 'physical' values $J=0,1,2 \ldots$. We would like to know this function on the real $J$-axis and in its vicinity. This would allow us to do the stationary phase analysis for trajectories, or to isolate contributions from a resonance poles, or to do both.

The $S$-matrix element is expected to have Regge poles, and also Regge zeroes 19 , i.e., the values of $J$ for which the transition $\nu \rightarrow \nu^{\prime}$ is impossible. Thus, it is reasonable to approximate $S_{\nu^{\prime} \leftarrow \nu}^{\alpha}(E, J)$ by a ratio of two polynomials, by constructing a Padé approximant

$$
S_{\nu^{\prime} \leftarrow \nu}^{P a d e}(E, J) \equiv K_{N} \exp \left[i\left(a J^{2}+b J+c\right)\right] \times \frac{\prod_{i=1}^{[N / 2]}\left(J-Z_{i}\right)}{\prod_{i=1}^{[(N-1) / 2]}\left(J-P_{i}\right)},
$$


which should have the correct values at the physical angular momenta,

$$
S_{\nu^{\prime} \leftarrow \nu}^{P a d e}(E, J)=S_{\nu^{\prime} \leftarrow \nu}^{\alpha}(E, J), \quad J=0,1, \ldots N .
$$

In equation $(6) K_{N}(E)$ is a constant, $[x]$ stands for the integral part of $x$, and $Z_{i}(E)$ and $P_{i}(E)$ are the zeroes and the poles of the approximant, respectively. There is also a quadratic phase specified by three constants, $a(E), b(E)$ and $c(E)$. Introduced to make the approximant more accurate, the phase has also a physical meaning: it is related to direct processes, which one should be able to describe in terms of trajectories. The construct in (6) is called Padé approximant of type II, to distinguish it from the approximant of type I, built from the values of the first $N$ derivatives at zero, rather than from $N$ values at different points 20 .

A method for constructing such approximants is described, for example, in Section III of Ref. 20. The method is implemented in the FORT AN code PADE_II 21 , whose purpose is to provide, as best one can, the values of the constants required to build $S_{\nu^{\prime} \leftarrow \nu}^{\text {Pade }}(E, J)$ in Eq.(6). For a detailed description of the code and the options availbale to the user, we refer the reader to Ref. 21. Here we only mention some of the properties of the function $S_{\nu^{\prime} \leftarrow \nu}^{\text {Pade }}$ built in this way from the values (5).

The fact that analytical continuation of the $S$-matrix element is not unique was noted, for example, in the early applications of the complex angular momentum (CAM) theory 22. One can, for example, multiply $S_{\nu^{\prime} \leftarrow \nu}^{\text {Pade }}(E, J)$ by $\exp (2 i m \pi J)$, where $m$ is any integer, and still satisfy Eqs.(7). Fortunately, in our semiclassical treatment this ambiguity can be resolved on physical grounds. With many angular momenta involved, the derivative of the phase of the $S$ matrix element, $d \Phi_{\nu^{\prime} \leftarrow \nu}^{\alpha}(E, J) / d J$, also known as the deflection function (DF), gives the angle into which products with angular momentum $J$ are scattered. If the forces between the atoms are mostly repulsive, and a particular choice of defining the angle is made, the $D F$ is expected to start at around $\pi$ for $J \approx 0$, and then descend to $\approx 0$ for $J \approx N$. The presence of resonances may complicate the form of the DF, but there is usually a way to check whether its overall shape complies with what has been just said. Another possibility is adding to $S_{\nu^{\prime} \leftarrow \nu}^{P a d e}$ a function which vanishes at $J=J_{\min }, \ldots, N$. This would change the behaviour of $\left|S_{\nu^{\prime} \leftarrow \nu}^{P a d e}\right|^{2}$ between, say, $J=5$ and $J=6$, where one expects it to be smooth. It is possible that a Regge pole very close to the real $J$-axis would give a narrow peak which fits between, say, $J=5$ and $J=6$. To check whether this resonance is indeed physical, one could change the energy until the peak is centred, say, at $J=6$ and becomes visible in the input data (5).

We note also that a Padé approximant, which has at its disposal roughly $N / 2$ poles, cannot correctly represent everywhere in the complex $J$-plane a $S_{\nu^{\prime} \leftarrow \nu}^{\alpha}(E, J)$ with an infinite number of poles. One might hope that at least $N / 2$ poles nearest to the real axis would be represented adequately. That is, unfortunately, not true. Some of the poles and zeroes of the approximant would indeed, coincide with the true physical poles and zeroes of $S_{\nu^{\prime} \leftarrow \nu}$. The rest will be scattered around, trying to mimic the effect of the remaining physical poles 
and zeroes for $J_{\mathrm{S}}$ close to the sampling values $J=J_{\text {min }}, \ldots N$. A calculation with a large number of partial waves $N$, and with few physical poles and zeroes close to the real axis, shows that most of the poles and zeroes form a closed boundary, within which the true poles and zeroes are reproduced accurately. Outside the boundary, the Padé approximant fails. One inexact yet helpful analogy is with trapping light between two concave mirrors, designed to give chosen values to the field on the central line between them. Inside the mirror cavity the light field is a smooth interpolation from the given values, while outside the field just vanishes, and no interpolation is produced. If the accuracy with which numerical data (5) was produced is poor, or the number of the partial waves is not sufficiently large, no well defined boundary is produced 14,17 . In this case, to check whether a pole is a physical one, or just an artefact of our Padé reconstruction, we should check its stability, e.g., by constructing approximants from different numbers of partial waves, or by contaminating the input data with more non-analytic noise 21.

Thus, after feeding in the data (5), and successfully running the PADE_II code, we have a function $S_{\nu^{\prime} \leftarrow \nu}^{\text {Pade }}(E, J)$ which correctly reproduces the chosen $S$-matrix element in a subset of the complex $J$-plane, containing the input points $J=$ $J_{\text {min }}, J_{\text {min }}+1, \ldots N$. With it, we can interpolate the $S$-matrix element between integer $J$ 's on the real $J$-axis, and evaluate $S_{\nu^{\prime} \leftarrow \nu}^{\alpha}$ for a complex $J$ not too far from it. We can find the positions $J_{n}=R e J_{n}+i \operatorname{Im} J_{n}$ of some of the true poles of the $S$-matrix, together with the corresponding residues, $\operatorname{Res}\left[S_{\nu^{\prime} \leftarrow \nu}^{\alpha}\left(E, J_{n}\right] \equiv\right.$ $\lim _{J \rightarrow J_{n}}\left(J-J_{n}\right) S_{\nu^{\prime} \leftarrow \nu}^{\alpha}\left(E, J_{n}\right)$. More specifically, we copute the residues as

$$
\operatorname{Res}\left[S_{\nu^{\prime} \leftarrow \nu}^{\alpha}\left(E, J_{n}\right)\right]=K_{N} \exp \left[i\left(a J_{n}^{2}+b J_{n}+c\right)\right] \frac{\prod_{i=1}^{[N / 2]}\left(J_{n}-Z_{i}\right)}{\prod_{i=1, i \neq j}^{[(N-1) / 2]}\left(J_{n}-P_{i}\right)} .
$$

Whatever we do, we may rely on this information only. In all applications we will be able to evaluate resonance contributions only for the genuine physical poles close to the real $J$ axis, whose positions and residues are known sufficiently accurately. The remaining poles will be used only to evaluate the $S$-matrix element only on the real axis, or close to it. In this way it doesn't matter if individual positions of those spurious poles are wrong, as long as together they produce a correct value for $S_{\nu^{\prime} \leftarrow \nu}^{\alpha}(E, J)$ where we need it.

\section{Understanding resonance patterns in state-to-state integral cross sections. The ICS_Regge package}

The first example, where the knowledge of the analytical properties of the $S$ matrix element can be used, concerns the integral cross section (2). An example of an ICS is shown in Fig. 2. What is the origin of the pattern seen there? We suspect it to be a resonance effect, yet Eq. (2) alone cannot provide a deeper insight, since the $S$-matrix elements contain both resonance and non-resonance contributions, and we do not know how to separate them. A more convenient 


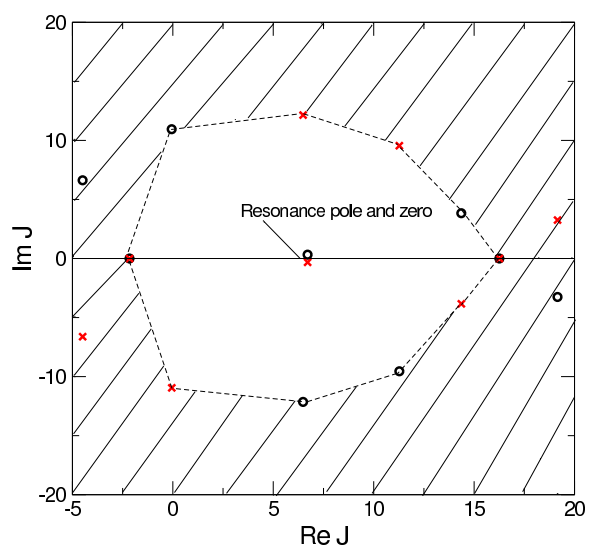

Fig. 1. Poles (circles) and zeroes (crosses) in the complex $J$-plane, as obtained by Padé reconstruction of the scattering matrix element. The potential model 12,14,15 consists of a hard sphere of a radius $2.045 \AA$ surrounded by semi-penetrable $\delta$-barrier of a magnitude $\Omega=1.023 \mathrm{meV} \cdot \stackrel{\AA}{A}$. at $r=$ $2.632 \AA$. The mass and the energy of the particle are 1 a.u. and $83.5 \mathrm{meV}$, respectively. There is one resonance Regge pole at $J_{1} \approx 6.71+0.32 i$, which corresponds to trapping the particle between the hard sphere and the outer layer. The Padé approximation uses 19 partial waves. It fails in the shaded region outside the boundary marked by the poles and zeroes of the approximant (dashed).

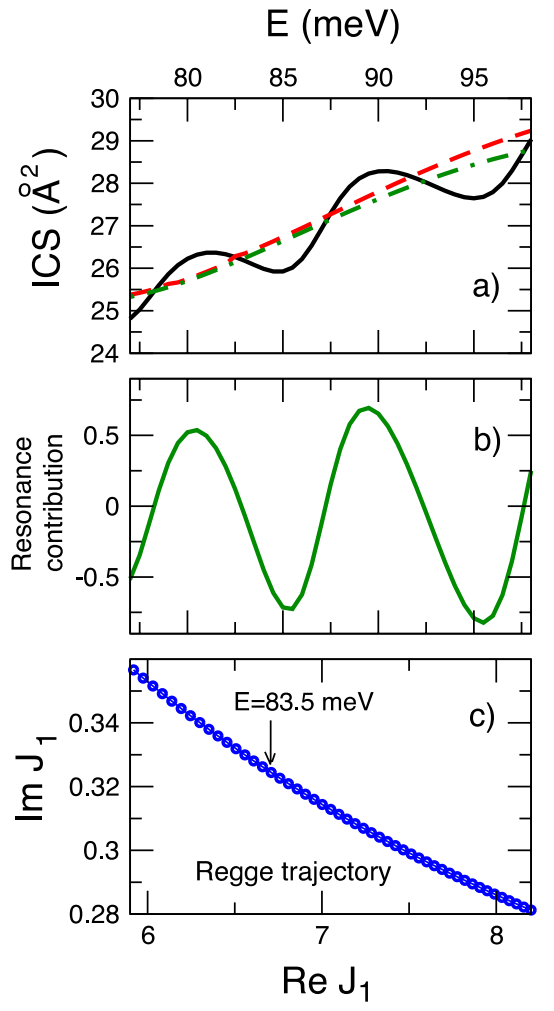

Fig. 2. a) The ICS for the model in Fig.1 (solid). Also shown are the first term in Eq.(9) (dashed) and the difference between the ICS and the resonance contribution in (b). b) the resonance contribution to the ICS given by the second term in the r.h.s of Eq.(9), and c) the corresponding resonance Regge trajectory. The arrow indicates the position of the resonance pole shown in Fig.1. 
representation was found in Ref. 23, and later modified in 24 to include state-tostate transitions in the multi-channel case. The Mulholland decomposition (we follow the authors of 23 in calling it so) can be obtained, for example by applying the Poisson sum formula to the sum in Eq. (2), and then deforming contours of integrations in the first and fourth quadrants in all but the zeroth terms. In the process we pick the contributions from all Regge poles, and are able to see which ones are responsible for the pattern in the ICS. As energy varies, Regge poles describe continuous curves in the complex $J$-plane, known as Regge trajectories. In fact, we are looking for Regge trajectories responsible for the pattern. The Mulholland formula, conventionally written in the variable

$$
\lambda \equiv J+1 / 2, \quad \lambda_{n}=J_{n}+1 / 2
$$

has the form

$$
\begin{gathered}
\sigma_{\nu^{\prime} \leftarrow \nu}^{\alpha}(E)=\frac{2 \pi}{k_{\nu}^{2}} \int_{J_{m i n}-1 / 2}^{\infty}\left|S_{\nu^{\prime} \leftarrow \nu}^{\alpha}(E, \lambda)\right|^{2} \lambda d \lambda+ \\
\frac{8 \pi^{2}}{k_{\nu}^{2}} \operatorname{Im} \sum_{n} \frac{\lambda_{n} \operatorname{Res}\left[S_{\nu^{\prime} \leftarrow \nu}^{\alpha}\left(E, \lambda_{n}\right)\right] S_{\nu^{\prime} \leftarrow \nu}^{\alpha *}\left(E, \lambda_{n}^{*}\right)}{1+\exp \left(-2 i \pi \lambda_{n}\right)}+I_{\nu^{\prime} \leftarrow \nu}(E),
\end{gathered}
$$

where a star denotes complex conjugation. The first term in (9) is what one gets by simply replacing the sum in (2) by an integral over $J$. It is what one would find if each angular momentum were scattered into its own angle, and to find the total number of scattered particles one only needs to sum over $J_{\mathrm{s}}$. The second term is the sum of all pole contributions, and reflects the ability of collision partners to form intermediate quasi-molecule(s). Thus, we expect it to be responsible for the resonance effects. The third term, $I_{\nu^{\prime} \leftarrow \nu}$ (, contains the remaining integrals. Their contours typically run up and down the imaginary $\lambda$-axis, where the exponentials in the Poisson sum formula decay rapidly. We expect $I_{\nu^{\prime} \leftarrow \nu}(E)$ to be small, or at least structureless 23,24. Since Eq.(9) is exact, this last term can be evaluated as the difference between the term in the 1.h.s and the first two terms in the r.h.s of Eq.(9)..

This is a formula which can be evaluated after performing Padé reconstruction described in the previous Section. The first term in the r.h.s of (9) can be computed, since from (6) we know the behaviour of $S_{\nu^{\prime} \leftarrow \nu}^{\alpha}$ on the real $J-$ $(\lambda-)$ axis. Although the sum in (9) runs over all poles in the first quadrant of the complex $J$-plane, only few of them contribute. Indeed, $1-\exp \left(-2 i \pi \lambda_{n}\right)=$ $1+\exp \left(-2 \pi R e J_{n}\right) \exp \left(2 \pi I m J_{n}\right)$ so that already for $I m J_{n}=1$ the denominator of order of $5 \times 10^{2}$ and, unless the residue is unduly large, the pole's contribution is negligible. Thus, it is the resonance poles close to the real axis that contribute most, and for such poles the Padé approximant gives accurate positions and residues. Finally, the Hermitian conjugate $S_{\nu^{\prime} \leftarrow \nu}^{\alpha *}\left(E, \lambda_{n}^{*}\right)$ is readily obtained by putting $J=J_{n}^{*}$ in Eq.(6), and taking conjugate of the result.

A suite of codes ICS_Regge 25 was recently produced for the purpose of identifying the relevant resonance contributions [the second term in Eq.(9)]. Again, here we only describe the main features of the code, and refer the reader to 
25 for details. The ICS_Regge employs a slightly modified (see 25) version of the PADE_II. For an input, one needs the numerical values of the $S$-matrix element, $S_{\nu^{\prime} \leftarrow \nu}^{\alpha}\left(E_{i}, J_{j}\right)$, evaluated on a grid of energies $E_{i}$, for a number of angular momentum $J_{j}$ sufficient to converge the sum (2) at each energy.

In the first step, a Padé reconstruction described in the previous Section, is performed for each energy $E_{i}$, and the Regge trajectories are identified.

In the second step, a particular trajectory is chosen and followed, with the resonance contribution evaluated at each energy. The result is subtracted from the exact ICS given by the PWS (2).

The second step can be repeated several times if there are several resonance trajectories which affect the ICS. After all resonances contributions have been removed, we are left with the 'direct' part of the cross section, often similar to the integral in Eq.(9).

An illustration is given in Fig. 2a, which shows the ICS for the model system used in Fig.1. What we learn is the following: there is an oscillatory structure seen in the ICS in the specified energy range. We want to know what has caused it. The Padé approximation is used to analytically continue the $S$-matrix element into the complex $J$ plane in the energy range of interest. In the region where the PD approximant is to be trusted, there is a Regge trajectory shown in Fig. 2c. As energy increases, the trajectory tends to approach the real axis, rather than to move away from it. This links the trajectory to a metastable, rather than a bound state of the potential 15 . Next we evaluate the contribution the trajectory makes to the full ICS. The contribution oscillates as is shown in Fig.2b, and when we subtract it from the ICS, the result is smooth and structureless. So the oscillations in Fig.2a are, indeed, caused by a single isolated resonance. This is a blueprint of how the code ICS_Regge can be used to analyse numerical data obtained for realistic reactive systems. For these much more complex systems one can encounter several, rather than just one, Regge trajectories. These trajectories can influence an ICS is in ways different from the one shown in Fig.2 15,23 , yet the method of the analysis remains the same.

One thing we haven't learnt yet is the physical origin of the resonance. To find out about that, we need to return to our model, and see that there is a possibility for a particle to penetrate the barrier, provided the angular momentum is such that its energy is close to the real part of the energy of a metastable state in the effective potential 15 . We can then identify the state in which the particle is trapped. Similarly, for a reactive system, the use ICS_Regge alone would not tell whether a resonance is caused by trapping the reactants in one of the Van der Waals wells, or help attribute particular quantum numbers to the state of the intermediate triatomic. This is, however,a sort of information usually available to someone who did the dynamical calculations to obtain the $S$-matrix elements numerically. In the end, we know the physical origin of a resonance, or resonances, and are able to quantify its or their effect on an ICS. This completes our analysis of the ICS, and leaves with the subject of angular distributions, also sensitive to the presence of resonances. 


\section{Resonance effects in state-to-state angular distributions. Future work}

Angular distributions, also known as differential cross sections (DCS), contain other detailed information of the reaction mechanism, and are more difficult to interpret. Although there has been much work on the analysis of DCSs in terms of trajectories and resonance poles 12, 13, 16, 14, 18, 19, 27, 26, no computer codes have yet been produced for public use. This Section is, therefore, more a statement of intent than a description of existing software.

To begin, there is a significant difference between transitions with zero and nonzero helicities 7. For $\Omega=\Omega^{\prime}=0$ the rotation matrix in Eq.(3) reduces to a Legendre polynomial of order $J$

$$
d_{00}^{J}(\cos \theta)=P_{J}(\cos \theta)
$$

This simplifies the analysis, and we assume this to be the case throughout this Section.

Consider again the simple case where a beam of particles of energy $E$ is scattered by a central potential. Classically, there are many trajectories with different impact parameters or, which is the same, with different angular momentum $J$. The scattering angle $\theta$ is between the initial and final velocities of the particle (see Fig.3). For our purpose, it is more convenient to look at the winding angle $\varphi$, by which the position vector of the particle rotates in the course of the collision. There may be a trajectory with $0<\varphi<\pi$, so the particle is just repelled by the potential. We will call such a trajectory 'first nearside (NS)'. Then there may also be a trajectory, with a different $J$, which does one full rotation around the origin, and exits with the same scattering angle, so that its winding angle is larger by $2 \pi$ (see Fig.3). We will label it 'second nearside'. Finally, a trajec-

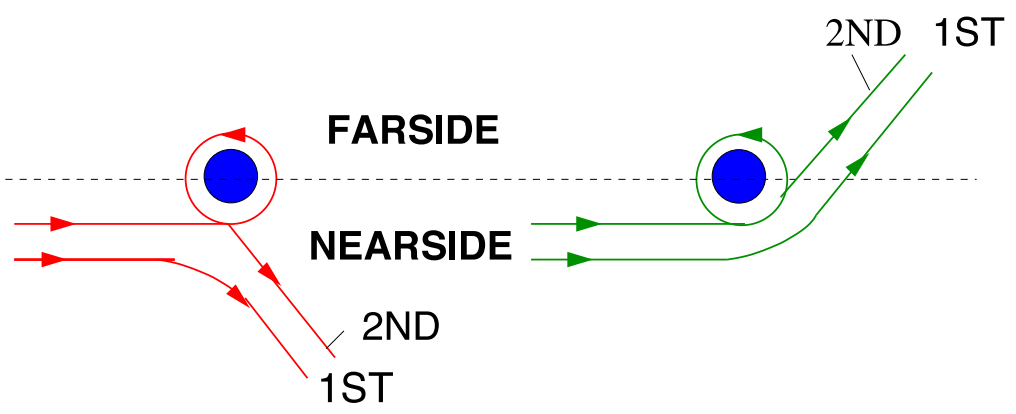

Fig. 3. A schematic diagram showing the first two nearside and farside trajectories.

tory undergoing $n-1$ full rotations well be called ' $n$-th nearside'. (Note that in Fig. 3 the angular momentum points towards the reader, so that the trajectories which enter as shown can only wind in the anti-clockwise sense, and $n$ is always 
positive.) There may be trajectories turning the corner, and exiting on the other side of the potential (see Fig.3). Their winding angles will be $\pi<\varphi<2 \pi$, plus multiples of $2 \pi$. If such trajectory makes $n-1$ full rotations, we will call it ' $n$-th farside (FS)'. Finally, due to axial symmetry, a NS trajectory with a winding angle $\varphi=\pi-\theta+2 \pi n, n=0,1,2 \ldots$, and a FS one with $\varphi=\pi+\theta+2 \pi m$, $m=0,1,2 \ldots$ correspond to the same scattering angle $\theta$.

Thus, there are, in principle, infinitely many ways in which a particle can arrive at a detector set at an angle $\theta$ to the direction of the beam. Classically, one simply counts the number of trajectories ending up between $\theta$ and $\theta+d \theta$. Quantally, each trajectory carries a probability amplitude, and these amplitudes are summed to obtain the scattering amplitude in Eq.(3). The types of trajectories, which can actually occur, depends on the potential. Let us assume that only the first NS and FS trajectories are present. Then there are just two pathways leading to a given $\theta$, and the system becomes a sort of two-arm interferometer, with an interference pattern revealed in the DCS $\sigma(\theta)$. This sets the rule of the game: we want to decompose a structured $f(\theta)$ into a sum of possibly structureless amplitudes, which also have some physical meaning. In our example these would be the contributions from the trajectories in Fig.3 which wind to the left and to the right. We may conclude that those which wind to the left pass through the attractive part of the potential, while the others are repelled by it.

There may be more trajectories, and we may not be interested in how many times they wind around the origin, just in whether they end up in the NS or the FS zone. Then we would combine the contributions from all NS and FS trajectories into $f^{N S}(\theta)$ and $f^{F S}(\theta)$, respectively, write $f(\theta)=f^{N S}(\theta)+f^{F S}(\theta)$, and see whether the interference between $f_{N S}$ and $f_{F S}$ is responsible for some interesting structure seen in $\sigma(\theta)$. As already mentioned, we would know nothing about the number of rotations a trajectory makes around the origin.

The picture involving classical trajectories was used for illustration only, and is by no means the only possible one. There are other, quantum, mechanisms making the particle circle around the origin. In the model used in Figs.1 and 2, a particle can tunnel through the outer barrier and find itself trapped. In order to conserve its angular momentum, such particle will have to spin around the hard core until it finally escapes. In a similar way, if three atoms form an intermediate triatomic, the triatomic must rotate before breaking into products, thereby carrying the atoms into angular regions not accessible to a direct scattering mechanism.

In a number of papers (see 28 and Refs. therein), Connor and co-workers have shown that, regardless of the physical mechanism, $f^{N S}(\theta)$ and $f^{F S}(\theta)$ can be evaluated simply by decomposing Legendre polynomials in (3) into two travelling wave components, $P_{J}(\cos \theta)=P_{J}^{+}(\cos \theta)+P_{J}^{-}(\cos \theta)$, where $P_{J}^{ \pm}(\cos \theta) \sim$ $\exp ( \pm i J \theta)$ provided $\theta$ is not too close to 0 or $\pi$. One then writes

$$
\begin{array}{r}
f_{\nu^{\prime} \leftarrow \nu}^{\alpha}(E, \theta)=\frac{1}{i k_{\nu}} \sum_{J=J_{\min }}^{\infty}(J+1 / 2)\left[P_{J}^{+}(\cos \theta)+P_{J}^{-}(\cos \theta)\right] \times \\
{\left[S_{\nu^{\prime} \leftarrow \nu}^{\alpha}(E, J)-\delta_{\alpha 0} \delta_{\nu \nu^{\prime}}\right] \equiv f_{\nu^{\prime} \leftarrow \nu}^{\alpha, N S}(E, \theta)+f_{\nu^{\prime} \leftarrow \nu}^{\alpha, F S}(E, \theta) .}
\end{array}
$$


The NF-decomposition (11) is extremely simple since it requires only the values of the $S$-matrix element at the physical values of $J, J=, 1,2 \ldots$, and no additional computational effort. Let us see what one can learn from it, using the simple model employed in Figs.1 and 2. The model is slightly modified: the contributions from higher angular momenta are now suppressed by multiplying the $S$-matrix element with a Gaussian, $S(E, J) \rightarrow \exp \left(-J^{2} / \beta^{2}\right) S(E, J)$, where $\beta$ is a suitably chosen constant. This helps us mimic a sort of a reaction, which is less likely to occur for large angular momenta, and also discard diffraction on the outer sphere, presently of no interest to us 14. The DCS shown in Fig.4a is highly structured, and we want to know the reason why it is so. The $f^{N S}(\theta)$ and $f^{F S}(\theta)$ in Eq.(11) are shown in Fig.4b. They are much less structured, so we find that most of the oscillations seen in Fig.3a come from the interference between the nearside and the farside components of the scattering amplitude, defined in Eq.(11).

We may want to know still more. There is still some structure left in the two components in Fig. 4b, and it is not quite clear yet what makes the system 'turn the corner', in order to produce the FS contribution in Fig.4a. More can be learned provided we know not just the discrete values of $S$, but its behaviour on the whole of the positive real $J$-axis. It was shown in 27 that it is sufficient to know just two functions,

$$
\begin{gathered}
F_{\nu^{\prime} \leftarrow \nu}^{\alpha}(E, \varphi)=\int_{0}^{\infty} \exp (i \lambda \varphi) S_{\nu^{\prime} \leftarrow \nu}^{\alpha}(E, \lambda) \lambda^{1 / 2} d \lambda \\
G_{\nu^{\prime} \leftarrow \nu}^{\alpha}(E, \varphi)=\int_{0}^{\infty} \exp (i \lambda \varphi) S_{\nu^{\prime} \leftarrow \nu}^{\alpha}(E, \lambda) \lambda d \lambda,
\end{gathered}
$$

in order to reconstruct the behaviour of the scattering amplitude $f_{\nu^{\prime} \leftarrow \nu}^{\alpha}(E, \theta)$ in the whole angular range $0 \leq \theta \leq \pi$. The details can be found in Ref 27 . Here we only note that by supplying more information, we also learn more: with the help of $F_{\nu^{\prime} \leftarrow \nu}^{\alpha}(E, \varphi)$ we 'unfold' the scattering amplitude not just into two NS and FS components, but into contributions coming from all NS and FS regions. An example of such detailed NF decomposition is given in Fig.5. There the unfolded amplitude $F$ shows a long decaying tail stretching into the 2-nd FS region, so that the structure left in the FS components in Fig. 4b comes from the interference between the 1-st and the 2-nd farside contributions. It is easy to verify that for $\varphi>>\pi, F_{\nu^{\prime} \leftarrow \nu}^{\alpha}(E, \varphi) \sim \operatorname{Res}\left[S_{\nu^{\prime} \leftarrow \nu}^{\alpha}\left(E, J_{1}\right)\right] \exp \left(i J_{1} \varphi\right)$, where $J_{1}$ is the position of the resonance Regge pole shown in Fig.1. Thus, the structure seen in Fig. 4a, results from capture into a resonance Regge state, in which the particle can perform up to two full rotations before finally escaping.

We can now outline the main tasks a code for analysing angular distributions (possibly called in future DCS_Regge) would need to perform:

a) analytically continue the $S$-matrix element into the complex $J$ plane using the PADE_II code and evaluate the functions $F$ and $G$ in Eq.(12) and all NS and FS contributions,

b) extract the resonance pole contributions from both $F$ and $G$, e.g., by the method described in 14, 26, 27 and express what is left in term of classical trajectories, 
c) using various contributions, reconstruct the DCS at various angles and for various energies,

d) explain, in physical terms, the shape of a DCS studied.

This will be the subject of our future work.

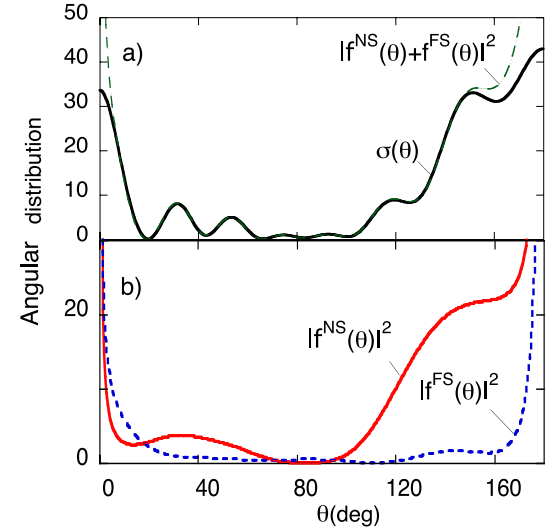

Fig. 4. a) The DCS for the model in Fig. 1 with $\beta=7$ (solid) and the sum of the nearside and farside components in Eq.(11) (dashed); b) the nearside and farside components in Eq.(11)

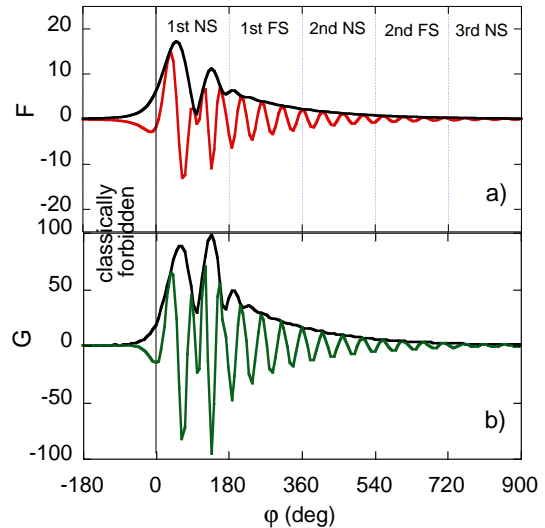

Fig. 5. The real parts and the moduli of the unfolded amplitude $F$ (a) and $G$ (b) given by Eq.(12) for the model in Fig.1. The region $\varphi<0$ is inaccessible to classical trajectories.

\section{The summary}

We argued that extracting full information about what happens in a chemical reaction is a task in its own right, which needs to be performed after the scattering matrix has been computed numerically. We have described the purpose of the computer codes, existing 21, 25 and yet to be produced, which would perform the analysis for the integral and differential cross sections. We use the language of resonance poles and/or classical trajectories where appropriate. Some of the theory required for the analysis already exists, while some aspects, such as angular scattering for non-zero helicities, would need further work. Our ultimate goal is to complement existing state-of-the-art reaction scattering codes 1-2 with additional numerical tools available to researchers working in the field.

\section{Acknowledgements:}

One of us (DS) acknowledges support of the Basque Government (Grant No. IT-472-10), and the Ministry of Science and Innovation of Spain (Grant No. FIS2009-12773-C02-01). The SGI/IZO-SGIker UPV/EHU is acknowledged for providing computational resources. 


\section{References}

1. D. Skouteris, J. F. Castillo and D. E. Manolopoulos, ABC: a quantum reactive scattering problem, Comp. Phys. Commun, 133 (2000) 128.

2. S. C. Althorpe, F. Fernández-Alonso, B. D. Bean, J. D. Ayers, A. E. Pomerantz, R. N. Zare and E. Wrede, Observation and interpretation of a time-delayed mechanism in hydrogen exchange reaction, Nature, 416 (2002) 67.

3. V. Aquilanti, S. Cavalli and D. De Fazio, Hyperquantization algorithm. I. Theory for triatomic systems, J. Chem. Phys., 109 (1998) 3792.

4. A. J. Alexander, D. A. Blunt, M. Brouard, J. P. Simons, F. J. Aoiz, L. Baares, Y. Fujimurac and M. Tsubouchic, $O\left({ }^{1} D_{2}\right)+H_{2} \rightarrow O H \mid v^{\prime} \leq 4, N^{\prime}>+H$ The anatomy of a reaction, Faraday Discuss., 108 (1997) 375.

5. W. H. Miller, Adv. Chem. Phys., Classical-Limit Quantum Mechanics and the Theory of Molecular Collisions, 25 (1974) 69.

6. W. H. Miller, Adv. Chem. Phys., The Semiclassical Initial Value Representation: A Potentially Practical Way for Adding Quantum Effects to Classical Molecular Dynamics Simulations, J. Phys. Chem., 105 (2001) 2942 .

7. D. Sokolovski and J.N.L Connor, Semiclassical nearsidefarside theory for inelastic and reactive atomdiatom collisions, Chem. Phys. Lett., 305 (1999) 238.

8. D. M. Brink, Semi-classical Methods in Nucleus-Nucleus Scattering, Cambridge University Press, Cambridge, 1985.

9. D. Sokolovski, S.K. Sen, V.Aquilanti, S.Cavalli and D.De Fazio, Interacting resonances in the $\mathrm{F}+\mathrm{H}_{2}$ reaction revisited: Complex terms, Riemann surfaces, and angular distributions J. Chem. Phys., 126 (2007) 084305.

10. For a review see J. N. L. Connor, J. Chem. Soc. Faraday Trans., 305 (1990) 1627.

11. D. Sokolovski, A.Z. Msezane, Z. Felfli, S.Yu. Ovchinnikov and J.H. Macek, What can one do with Regge poles?, Nuclear Instruments and Methods in Physics Research Section B: Beam Interactions with Materials and Atoms, Volume 261 (2007) 133.

12. D. Sokolovski, Complex-angular-momentum (CAM) route to reactive scattering resonances: from a simple model to the $\mathrm{F}+\mathrm{H}_{2} \rightarrow \mathrm{HF}+\mathrm{H}$ reaction , Phys. Scr., 78 (2008) 058118.

13. J. N. L. Connor, Resonance Regge poles and the state-to-state $\mathrm{F}+\mathrm{H} 2$ reaction: QP decomposition, parametrized S matrix, and semiclassical complex angular momentum analysis of the angular scattering, J. Chem. Phys. 138124310 (2013).

14. D. Sokolovski and A. Z. Msezane, Semiclassical complex angular momentum theory and Padé reconstruction for resonances, rainbows, and reaction thresholds, Phys. Rev. A., 70 (2004) 032710.

15. D. Sokolovski and E. Akhmatskaya, Phys. Lett. A, 375 (2011) 3062.

Please note that there is a typo in Eq.(5), which should read

$$
\begin{gathered}
S(k, \lambda)=\Delta^{(2)}(E, \lambda) / \Delta^{(1)}(E, \lambda) \equiv \\
-\left.\frac{H_{\lambda}^{(2)}(k r)\left\{\left[\ln H_{\lambda}^{(2)}(k r)\right]^{\prime}-\left[\ln \phi_{\text {well }}(r, k, \lambda)\right]^{\prime}-2 \Omega\right\}}{H_{\lambda}^{(1)}(k r)\left\{\left[\ln H_{\lambda}^{(1)}(k r)\right]^{\prime}-\left[\ln \phi_{\text {well }}(r, k, \lambda)\right]^{\prime}-2 \Omega\right\}}\right|_{r=R},
\end{gathered}
$$

with $\phi(r)=A(k, \lambda) \sqrt{r} \phi_{\text {well }}(r)$ and $q \equiv\left(k^{2}+2 V\right)^{1 / 2}$.

16. C. Xiahou, J.N.L Connor and D. H. Zhang, Rainbows and glories in the angular scattering of the state-to-state $\mathrm{F}+\mathrm{H}_{2}$ reaction at $E_{\text {trans }}=0.04088 \mathrm{eV}$, Phys. Chem. Chem. Phys., 13, (2011), 12981.

17. D. Sokolovski, Glory and thresholds effects in $\mathrm{H}+\mathrm{D}_{2}$ reactive angular scattering, Chem. Phys. Lett., 370 (2003) 805. 
18. C. Xiahou and J.N.L Connor, The 6 Hankel asymptotic approximation for the uniform description of rainbows and glories in the angular scattering of state-to-state chemical reactions: derivation, properties and applications, Phys. Chem. Chem. Phys., (2014) (in print)

19. D. Sokolovski, Complex-angular-momentum analysis of atom-diatom angular scattering: Zeros and poles of the S matrix, Phys. Rev. A., 62 (2000) 024702-01.

20. D. Bessis, A. Haffad, and A. Z. Msezane, Momentum-transfer dispersion relations for electron-atom cross sections, Phys. Rev. A., 49 (1994) 3366.

21. D. Sokolovski, E. Akhmatskaya and S. K. Sen, Extracting resonance poles from numerical scattering data: type-II Padé reconstruction, Comp. Phys. Comm. A, 182 (2011) 448.

22. T. Regge, Nuovo Cimento, 14 (1959) 947.

23. J. H. Macek, P. S. Krstic, and S. Yu. Ovchinnikov, Regge oscillations in integral cross sections for proton impact on atomic hydrogen, Phys. Rev. Lett. 93 (2004) 183203.

24. D. Sokolovski, D.De Fazio, S.Cavalli and V.Aquilanti, Overlapping resonances and Regge oscillations in the state-to-state integral cross sections of the $\mathrm{F}+\mathrm{H}_{2}$ reaction, J. Chem. Phys., 126 (2007) 12110.

25. E. Akhmatskaya, D. Sokolovski and C. Echeverría-Arrondo, Numerical Regge pole analysis of resonance structures in elastic, inelastic and reactive state-to-state integral cross sections, Comp. Phys. Comm. A, (2014) (in print).

26. D. Sokolovski, S.K. Sen, V.Aquilanti, S.Cavalli and D.De Fazio, Interacting resonances in the $\mathrm{F}+\mathrm{H}_{2}$ reaction revisited: Complex terms, Riemann surfaces, and angular distributions J. Chem. Phys., 126 (2007) 084305.

27. D. Sokolovski, D.De Fazio, S.Cavalli and V.Aquilanti, On the origin of the forward peak and backward oscillations in the the $\mathrm{F}+\mathrm{H}_{2}(\mathrm{v}=0) \rightarrow \mathrm{HF}\left(\mathrm{v}^{\prime}=2\right)+\mathrm{H}$ reaction, Phys.Chem.Chem.Phys., 9 (2007) 1.

28. A.J. Dobbyn, P. McCabe, J.N.L Connor and J.F. Castillo, Nearside-farside analysis of state-selected differential cross sections for reactive molecular collisions, Phys. Chem. Chem. Phys., 1 (1999) 1115. 